\title{
Effects of an Enteral Formula Containing Fermented Dairy Products on Epithelial Ion Transport in Rat Intestines
}

\author{
Shin-Ichiro KARAKI \\ Laboratory of Physiology, Department of Environmental and Life Sciences, School of Food and Nutritional \\ Sciences, University of Shizuoka, 52-1 Yada, Suruga-ku, Shizuoka 422-8526, Japan
}

(Received March 12, 2019)

\begin{abstract}
Summary Diarrhea is the most common complication of enteral nutrition (EN). Pro/prebiotics are typically used to prevent diarrhea during EN. This study aimed to demonstrate the effects of enteral formula containing fermented dairy products (FDPs) and galacto-oligosaccharides on intestinal mucosal functions in rats. After feeding rats with regular rodent chow (RRC), standard formula (STD-F), and FDP-containing formula (FDP-F) for $2 \mathrm{wk}$, the rats were sacrificed with their intestines removed. Then, the electrophysiological properties of intestinal epithelia were measured using the Ussing chamber. In addition, organic acids and microbiota in the cecal contents were analyzed. In FDP-F-fed rats, electrical nerve activation-evoked increase in short-circuit current $\left(I_{\mathrm{sc}}\right)$ in the cecum and middle colon was reduced compared with STD-F-fed rats. Mucosal propionate-evoked changes in $I_{\mathrm{sc}}$ in FDP-F-fed rats were also reduced in the terminal ileum. The total cecal organic acid concentration in STD-F-fed rats decreased compared with RRC-fed rats, and approximately half was recovered in FDP-F-fed rats, which contributed to the recovery of acetate and butyrate concentrations. In microbiota analysis, the density of total bacteria, particularly Bifidobacterium, in cecal contents increased in FDP-F-fed rats. In conclusion, the consumption of FDP-F changed the total amounts and components of gut microbiota and organic acids, and resulted in inhibitory changes in mucosal luminal stimulant- and nervous system-mediated fluid secretory function. These findings suggest that FDP-F might prevent the incidence of diarrhea during EN.
\end{abstract}

Key Words enteral nutrition, diarrhea, probiotics, prebiotics, short-chain fatty acids, Ussing chamber

Enteral nutrition (EN) provides nutrients directly into the gastrointestinal (GI) tract bypassing the oral cavity. It is required for patients with swallowing or chewing problems in the hospital or community setting. EN is a physiological feeding associated with fewer serious complications compared with parenteral nutrition (central venous feeding). However, complications, such as nausea, bloating, diarrhea, excessive ostomy output, constipation, abdominal discomfort or pain, and reflux, often occur during EN (1). In particular, diarrhea is the most common complication of $\mathrm{EN}$; it affects $2 \%$ to $95 \%$ (this wide range resulted from the differences in the patient groups investigated and variations in the definition of diarrhea used) of patients (2).

Several causes of diarrhea during EN, including malabsorption, infection, bacterial contamination of diet, medical diagnosis of the patient, medication therapy, and formula-related causes, are considered (3). GI microbiota and short-chain fatty acids (SCFAs), which are fermentation products of GI microbiota, are implicated in the incidence of diarrhea during EN (4). Therefore, the roles of probiotics, defined as "live microorganisms which when administered in adequate amounts confer a health benefit on the host," (5) and prebiot-

E-mail:karaki@u-shizuoka-ken.ac.jp ics, defined as "selectively fermented ingredients that allow specific changes, both in the composition and/or activity in the gastrointestinal microflora that confers benefits upon host well-being and health," (6) in the reduction of the incidence of diarrhea during EN have been investigated $(2,7)$. Probiotics, such as Lactobacillus rhamnosus GG, have not shown promise as a treatment for diarrhea that is already occurring in critically ill patients (8). However, Saccharomyces boulardii as a probiotic has been reported to prevent diarrhea in critically ill patients (9). In a meta-analysis study of prebiotics, fiber-supplemented enteral formula (EF) has been found to have clinical benefit in reducing the incidence of diarrhea (10), but fructo-oligosaccharides (FOS), oligofructose, and inulin (as prebiotics) supplementations in EF did not minimize the incidence of diarrhea in EN $(11,12)$.

Fermented milk by lactic acid bacteria (LAB) is the first probiotic advocated by Metchnikoff in 1908 (13). Therefore, LAB-fermented milk has prospect of beneficial effects on diarrhea during EN. LAB-fermented milkcontaining EF is at least clinically speculated to prevent the incidence of diarrhea; however, its mechanism is still unclear.

The fermented dairy product-containing formula (FDP-F) in the present study contained LAB (Lactobacil- 
lus bulgaricus and Streptococcus thermophilus)-fermented milk, propionic acid bacteria (PAB) (Propionibacterium freudenreichii ET-3)-fermented milk whey, and galactooligosaccharides (GOS). L. bulgaricus and S. thermophilus are the traditionally used combination for the production of yoghurt (14). PAB-fermented milk whey has a prebiotic function that enhances the growth of Bifidobacterium in normal adult humans (15), and 1, 4-dihydroxy-2-naphthoic acid is isolated and identified as a bifidogenic growth stimulator (16).

Infusion of SCFAs directly into the cecum has been reported to reverse the fluid secretion found in the ascending colon during EN (17). We therefore hypothesized that FDP-F-feeding may change the GI microbiota, increase SCFAs in the large intestinal contents, and induce an inhibitory effect on the secretory function of epithelia. Thus, in the present study, we aimed to investigate the effects of the consumption of sterilized FDP-F on the transepithelial ion transport inducing fluid secretion or absorption in the rat small and large intestines.

In the present study, fluid secretion in the intestinal epithelia was measured by Ussing chamber technique as an electrophysiological method (18). Short-circuit current $\left(I_{\mathrm{sc}}\right)$ measured by the Ussing chamber technique is an index of net transepithelial ion transport. Driving force of intestinal fluid secretion is produced by the increase in luminal osmotic pressure. Active and electrogenic anion $\left(\mathrm{Cl}^{-}\right.$and $\left.\mathrm{HCO}_{3}{ }^{-}\right)$transport from serosal to luminal side of the epithelium produce a lumen negative potential difference, which induces passive cation $\left(\mathrm{Na}^{+}\right)$secretion and then increases luminal $\mathrm{NaCl}$ concentration and osmotic pressure. Therefore, measurement of the electrogenic anion secretion by Ussing chamber technique as a change in $\Delta I_{\mathrm{sc}}$ is relevant for evoking a massive fluid secretion and diarrhea. In addition, the epithelial sodium channel (ENaC) expressed in the apical membrane especially in the distal colonic and rectal epithelia induces an electrogenic $\mathrm{Na}^{+}$absorption that increases $I_{\mathrm{sc}}$, and potently absorbs luminal water. Therefore, the inhibition of $\mathrm{ENaC}$ activity that decreases $I_{\mathrm{sc}}$ induces an inhibition of water absorption in the distal colon and rectum, possibly inducing diarrhea. These indicate that both increase and decrease in $I_{\mathrm{sc}}$ possibly induces diarrhea. Therefore, in the present study, measurements of the changes in $I_{\mathrm{sc}}$ were performed to investigate the effects of the consumption of FDP-F.

\section{MATERIALS AND METHODS}

Experimental diets. Powder diets of two freeze-dried formulas and one regular rodent chow (RRC) were used. Meibalance (Meiji, Tokyo, Japan) as a standard formula (STD-F), YH Flore (Meiji) as an FDP-F, and CRF-1 (Oriental Yeast Co., Ltd., Tokyo, Japan) as an RRC were used. YH Flore, but not Meibalance, contained sterilized LAB (L. bulgaricus and S. thermophilus)-fermented milk, sterilized PAB ( $P$. freudenreichii ET-3)-fermented milk whey (Profec $^{\circledR}$, Meiji), and GOS. The components of these experimental diets are shown in Table S1 (Supplemental Online Material). These enteral formulas were kindly gifted by Meiji Co., Ltd.
Animals. The animals were handled and killed in accordance with the Guidelines for the Care and Use of Laboratory Animals of the University of Shizuoka, and the study was approved by the University of Shizuoka Animal Usage Ethics Committee (approval No. 145069 and 1551103 approved on 11 August, 2014 and 9 September, 2015, respectively). Male Sprague-Dawley rats aged $6 \mathrm{wk}(n=18)$ were purchased from Japan SLC, Inc. (Hamamatsu, Japan). After a 1-wk acclimatization period, they were divided into three groups (six rats per group) by body weight-stratified randomization: RRC-, STD-F-, and FDP-F-fed groups. The animals were fed with each diet and given water ad libitum for 2 wk. In the present study, food intake could not be measured because the powder diet was spilled during the feeding and the metabolic cages were not used.

Sampling and tissue preparation for the Ussing chamber experiments measuring the electrogenic transepithelial ion transport. Two-week RRC-, STD-F-, and FDP-Ffed rats were anesthetized with isoflurane inhalation and decapitated with a guillotine. The GI tract en bloc from the stomach to the anus was removed and then immersed in ice-cold Krebs-Ringer solution containing (mM) $117 \mathrm{NaCl}, 4.7 \mathrm{KCl}, 1.2 \mathrm{MgCl}_{2}, 1.2 \mathrm{NaH}_{2} \mathrm{PO}_{4}$, $25 \mathrm{NaHCO}_{3}$, and $2.5 \mathrm{CaCl}_{2}$. The solution was saturated with $95 \% \mathrm{O}_{2}-5 \% \mathrm{CO}_{2}$. Mesenteric and fat tissues were removed from the specimens, and the isolated GI tracts were extended on a Ringer-soaked paper towel. Photo images were taken with a ruler using a digital camera to measure the length of the intestine. During the middle of experiments, I found that the size of the cecum was very different among the experimental groups. Therefore, I added the analysis of cecal tissue and the contents in residual 3 animals from that time. The cecum was removed, and gross weight (cecal tissue + contents) was measured. The cecum was cut open along the mesentery of the lesser curvature, and the cecal contents were taken to measure the organic acid concentrations. The contents were washed out, and cecal tissue weight was measured. The cecal content samples were frozen and stored at $-80^{\circ} \mathrm{C}$ until analysis.

For the Ussing chamber experiments, the terminal ileum, cecum, proximal colon, middle colon, and distal colon were removed, cut open along the mesenteric border, and pinned flat to the silicon-rubber-lined petri dish with Krebs-Ringer solution. To investigate the electrophysiological properties of the epithelium, mucosasubmucosa preparations were made by removing the muscle layer under stereomicroscopy.

Ussing chamber experiments. Ussing chamber experiments were performed following a method described previously (19). Mucosa-submucosa preparations were mounted on the Ussing chambers (cross-sectional area: $0.64 \mathrm{~cm}^{2}$; CHM2, World Precision Instruments (WPI), Inc., Sarasota, FL, USA) with a pair of aluminum foil ribbon electrodes for electrical field stimulation (EFS). Both sides were perfused with $10 \mathrm{~mL}$ of Krebs-Ringer solution each by recirculation from the glass circulation reservoir (\#5210, WPI) maintained at $37^{\circ} \mathrm{C}$ and gassed with $95 \% \mathrm{O}_{2}-5 \% \mathrm{CO}_{2}$. D-Glucose $(5 \mathrm{mM})$ was added 
Table 1. Body weight, lengths of intestines, and cecal conditions in RRC-, STD-F- and FDP-F-fed rats.

\begin{tabular}{lccc}
\hline & RRC & STD-F & FDP-F \\
\hline Body weight $(\mathrm{g}), n=6$ & $319.9 \pm 8.1$ & $308.2 \pm 8.1$ & $305.7 \pm 7.8$ \\
Length of small intestine $(\mathrm{mm}), n=6$ & $693.4 \pm 40.4$ & $592.3 \pm 42.1$ & $613.5 \pm 45.3$ \\
Length of large intestine $(\mathrm{mm}), n=6$ & $152.7 \pm 6.5^{\mathrm{a}}$ & $126.1 \pm 6.9^{\mathrm{b}}$ & $135.9 \pm 4.6^{\mathrm{ab}}$ \\
Cecal tissue weight $(\mathrm{g}), n=3$ & $1.13 \pm 0.09^{\mathrm{a}}$ & $1.12 \pm 0.10^{\mathrm{a}}$ & $1.69 \pm 0.12^{\mathrm{b}}$ \\
Wet weight of cecal contents $(\mathrm{g}), n=3$ & $5.50 \pm 0.03^{\mathrm{a}}$ & $3.66 \pm 0.43^{\mathrm{b}}$ & $4.49 \pm 0.33^{\mathrm{ab}}$ \\
\hline
\end{tabular}

Data are expressed as mean \pm SE. Different superscript alphabets differ significantly $(p<0.05)$ by Tukey test.

Table 2. Basal electrophysiological parameters of the mucosa-submucosa preparations in a variety of intestinal segments in RRC-, STD-F- and FDP-F-fed rats.

\begin{tabular}{lcrr}
\hline & RRC & STD-F & FDP-F \\
\hline${\text { Basal } I_{\text {sc }}\left[\mu \mathrm{A} / \mathrm{cm}^{2}\right]}$ & & & $13.21 \pm 3.86$ \\
Terminal ileum & $17.95 \pm 2.25$ & $30.95 \pm 9.37$ & $8.76 \pm 3.36$ \\
Cecum & $21.84 \pm 6.62$ & $32.77 \pm 11.17$ & $34.63 \pm 2.91$ \\
Proximal colon & $30.80 \pm 3.97$ & $35.73 \pm 1.86$ & $21.22 \pm 3.66$ \\
Middle colon & $16.06 \pm 0.58$ & $17.28 \pm 1.29$ & $20.62 \pm 3.21$ \\
Distal colon & $13.29 \pm 2.33$ & $21.44 \pm 5.41$ & $1.38 \pm 3.68$ \\
Rectum & $3.25 \pm 1.78$ & $12.42 \pm 5.16$ & $21.52 \pm 0.53$ \\
Basal $G_{\mathrm{t}}\left[\mathrm{mS} / \mathrm{cm}^{2}\right]$ & $20.12 \pm 0.44$ & $19.46 \pm 0.80$ & $25.82 \pm 2.74^{\mathrm{b}}$ \\
Terminal ileum & $13.63 \pm 0.99^{\mathrm{a}}$ & $13.57 \pm 0.78^{\mathrm{a}}$ & $22.03 \pm 3.38$ \\
Cecum & $20.27 \pm 1.07$ & $15.22 \pm 1.51$ & $29.53 \pm 4.94^{\mathrm{b}}$ \\
Proximal colon & $13.42 \pm 1.96^{\mathrm{a}}$ & $13.50 \pm 1.41^{\mathrm{a}}$ & $13.03 \pm 1.29^{\mathrm{b}}$ \\
Middle colon & $7.58 \pm 0.83^{\mathrm{a}}$ & $12.37 \pm 2.05^{\mathrm{a}, \#}$ & $10.63 \pm 1.68$ \\
Distal colon & $7.82 \pm 1.22$ & $17.15 \pm 3.45^{\#}$ & \\
Rectum & & & \\
\hline
\end{tabular}

Data are expressed as mean \pm SE $(n=4-6)$. Different superscript alphabets differ significantly $(p<0.05)$, and \# indicates tendency $(p<0.10)$ of difference vs RRC by Tukey test (distal colon) or Games-Howells test (cecum, middle colon and rectum).

to the serosal solution only. The transepithelial potential difference (the serosal electrode served as the reference) was detected by paired $\mathrm{Ag}-\mathrm{AgCl}$ electrodes (EKV, WPI) through Krebs-agar bridges, and was clamped at $0 \mathrm{mV}$ by applying an $\mathrm{I}_{\mathrm{sc}}$ with another pair of $\mathrm{Ag}-\mathrm{AgCl}$ electrodes (EKC, WPI) connecting a voltage-clamp apparatus (CEZ-9100; Nihon-Kohden, Tokyo, Japan). The lumen negative electrogenic current indicates a value of positive $I_{\mathrm{sc}}$ per unit area of tissue $\left(\mu \mathrm{A} / \mathrm{cm}^{2}\right)$. To record tissue conductance $\left(G_{\mathrm{t}}\left[\mathrm{mS} / \mathrm{cm}^{2}\right]\right)$, voltage command pulses (10 mV, 3-s duration) were applied at 1-min intervals, and $G_{\mathrm{t}}$ was calculated by Ohm's law from necessary to current necessary to change the clamped voltage. The current output was continuously recorded on a data acquisition and analog-to-digital conversion system (PowerLab 4/26; ADInstruments, Cattle Hill, Australia). Immediately after mounting the tissues, $I_{\mathrm{sc}}$ and $G_{t}$ showed higher value, then gradually decreased, and achieved plateau within $1 \mathrm{~h}$ (Fig. 1). Thus, tissues were stabilized for $1 \mathrm{~h}$ before the experiments.

Protocol of the Ussing chamber experiments. After a 1-h stabilization period, basal $I_{\mathrm{sc}}$ and basal $G_{\mathrm{t}}$ were determined, and the tissues were electrically stimulated at $25 \mathrm{~V}, 5 \mathrm{~Hz}, 0.5 \mathrm{~ms}$-duration for $2 \mathrm{~min}$ via a pair of aluminum foil ribbon electrodes. Propionate (1 mM) was added to the mucosal bathing solution $1 \mathrm{~h}$ after the EFS, and changes in $I_{\mathrm{sc}}$ were measured. Further $1 \mathrm{~h}$ after the addition of propionate, carbachol $(\mathrm{CCh})$ was added to the serosal bathing solution. Representative $I_{\mathrm{sc}}$ traces of the RRC-fed group from the time immediately after mounting tissues to the time after the final addition of CCh are shown in Fig. 1.

Chemicals. Sodium propionate was purchased from Wako Pure Chemical Industries, Ltd. (Osaka, Japan), and CCh was obtained from Sigma (St. Louis, MO, USA).

Organic acid concentrations in the cecal contents. Approximately $200 \mathrm{mg}$ of cecal content was diluted with 2 volumes of Milli-Q water and homogenized, followed by centrifugation for $10,000 \mathrm{rpm}$ at $4^{\circ} \mathrm{C}$ for $10 \mathrm{~min}$. About $2.5 \mu \mathrm{L}$ of Carrez Reagent I (53.5 g $\mathrm{ZnSO}_{4} \cdot 7 \mathrm{H}_{2} \mathrm{O} / 100 \mathrm{~mL}$ Milli-Q) and $2.5 \mu \mathrm{L}$ of Carrez Reagent II [17.2 g K $\mathrm{K}_{4} \mathrm{Fe}(\mathrm{CN})_{6} \cdot 3 \mathrm{H}_{2} \mathrm{O} / 100 \mathrm{~mL}$ Milli-Q] were added to $200 \mu \mathrm{L}$ of the supernatant to precipitate protein (20). The supernatant was passed through a $0.22-\mu \mathrm{m}$ filter and used for the assay. Organic acids were measured with a post column $\mathrm{pH}$ buffering electric conductivity detection method using an electric conductivity detector (CDD-10A, Shimadzu Corporation) according to the modified method of Niwa et al. (21). A double-connected polymer column for organic acid 

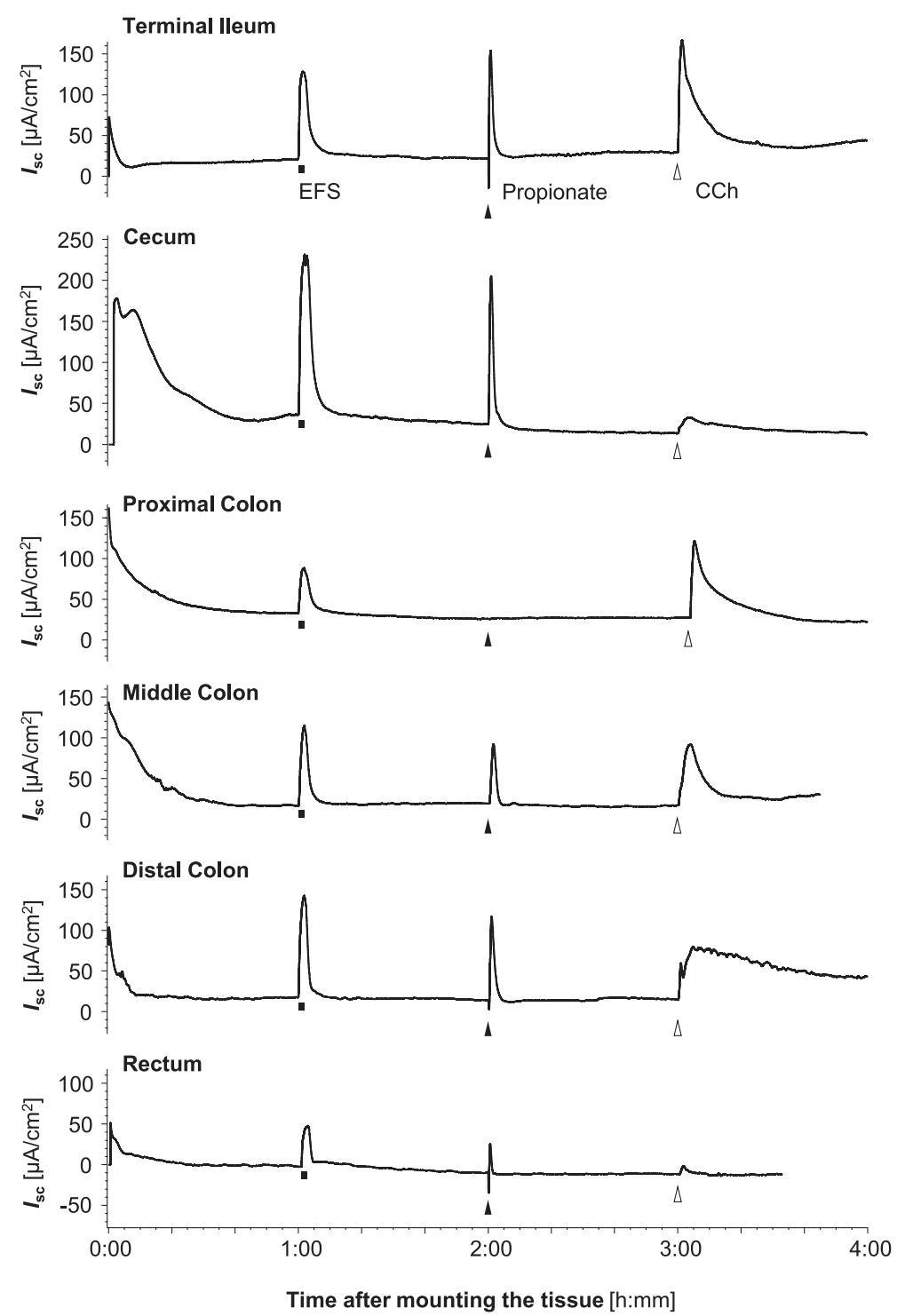

Fig. 1. Representative $I_{\mathrm{sc}}$ traces of each intestinal segment of the mucosa-submucosal tissues isolated from an RRC-fed rat from start to end of the experiment. Mucosa-submucosal tissues of terminal ileum, cecum, proximal colon, middle colon, distal colon and rectum were mounted on the Ussing chambers, and $I_{\mathrm{sc}} \mathrm{S}$ were continuously recorded. One hour after the mounting the tissues for stabilization, the tissues were electrically stimulated ( $\mathbf{\square}, 25 \mathrm{~V}, 5 \mathrm{~Hz}, 0.5 \mathrm{mS}$-duration, for $2 \mathrm{~min}$ ).

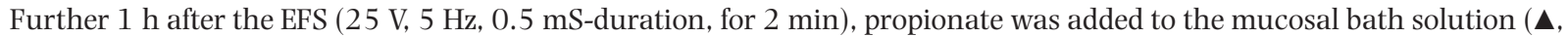
bath concentration: $10^{-3} \mathrm{M}$ ). Finally, $\mathrm{CCh}$ was added to the serosal bath solution ( $\triangle$, bath concentration: $\left.10^{-5} \mathrm{M}\right) 1 \mathrm{~h}$ after the addition of propionate.

analysis (ICSep-ORH-801 $6.5 \mathrm{~mm} \times 300 \mathrm{~mm}$; Tokyo Chemical Industry Co., Ltd.) was used at a column temperature of $50^{\circ} \mathrm{C}$, mobile phase of $5 \mathrm{~mm}$-trinitrosulfonic acid solution, reaction solution of $20 \mathrm{~mm}$ Bis-Tris aqueous solution containing $5 \mathrm{mM}$ p-trinitrosulfonic acid solution and $100 \mu \mathrm{M}$ EDTA $(2 \mathrm{Na})$, and flow rate of $0.5 \mathrm{~mL} / \mathrm{min}$. The total concentration was calculated as the sum of each organic acid.

Analysis of microbiota. Analysis of microbiota in the cecal contents was performed using the same method previously reported $(22,23)$. The microbiota DNA in the cecal content samples was extracted using QIAamp DNA stool mini kit (QIAGEN, Tokyo, Japan) and multi-beads shocker (Yasui Kikai, Osaka, Japan). Counts of total and individual bacterium [Bifidobacterium (24), Lactobacillus
(25), Bacteroides (24), Clostridium coccoides (24), Clostridium leptum (24), Enterobacteriaceae (26), Enterococcus (27)] were determined with the ABI7300 real-time PCR system (Applied Biosystems, Tokyo, Japan) using QuantiTect SYBR Green RT-PCR (QIAGEN) and published primer base sequences and reaction conditions. The numbers of total bacteria and individual bacterium per gram of wet cecal contents were determined.

Statistics. Data are expressed as mean \pm SE after outlier values were removed by Smirnov-Grubbs test. Tukey-HSD test in homoscedasticity and Games-Howells test in heteroscedasticity were used to determine statistically significant difference $(p<0.05)$ and tendency $(0.05 \leq p<0.10)$ between each group using SPSS Statistics version 23 (IBM, Armonk, NY, USA). 


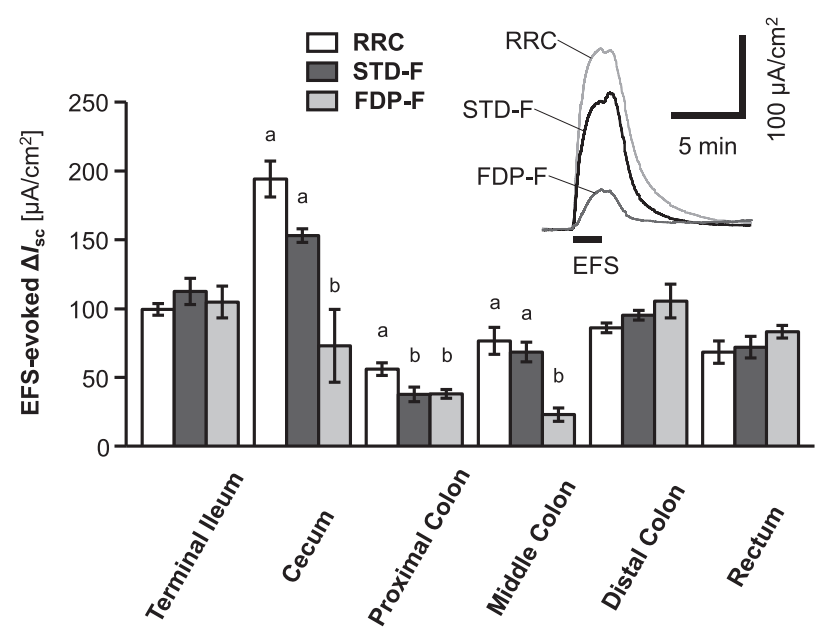

Fig. 2. Effects of RRC, STD-F and FDP-F feedings on the EFS-evoked increase in $I_{\mathrm{sc}}$ in mucosa-submucosal preparations of each segment of rat intestines. Tissues were electrically stimulated at $25 \mathrm{~V}, 5 \mathrm{~Hz}$ and $0.5 \mathrm{mS}$-duration for $2 \mathrm{~min} 1 \mathrm{~h}$ after mounting, and the EFS-evoked increase in $I_{\mathrm{sc}}$ was determined. Data were expressed as mean \pm SE $(n=4-6)$. Different superscript alphabets differ significantly $(p<0.05)$ by Tukey-HSD test. Inset traces: Stacks of representative traces of EFS-evoked $\Delta I_{\text {sc }}$ in each groups in the cecum.

\section{RESULTS}

Body weight, lengths of the small and large intestines, and weights of cecal tissue and its content

Data on body weight, lengths of intestines, and cecal conditions are shown in Table 1 . The body weight and length of the small intestine (from the pyloric sphincter to the ileocecal junction) were not significantly different between each diet-fed group. However, the length of the large intestine (from the cecocolonic junction to the anus) in the STD-F-fed group was significantly shorter than that in the RRC-fed group. In the FDP-F-fed group, the length of the large intestine was not significantly different from the RRC- or STD-F-fed group.

The wet cecal tissue weight in the FDP-F-fed group was significantly heavier than that in the RRC- and STDF-fed groups. Moreover, the wet weight of cecal contents in the STD-F-fed rats was significantly lighter than that in the RRC-fed group, but not than that in the FDP-F-fed group. The contents of the large intestine in the FDP-Ffed group seemed to be stickier in consistency than that in the other groups.

Basal electrophysiological parameters in the intestinal mucosa-submucosa preparations

Basal $I_{\mathrm{sc}}$ and $G_{\mathrm{t}}$ in mucosa-submucosal tissue preparations from each diet-fed rat are shown in Table 2. In basal $I_{\text {sc }}$, no significant difference and tendency were found between each other. In basal $G_{t}$, the values of the cecum, middle colon, and distal colon isolated from the FDP-F-fed group were significantly higher than those from the other diet groups. Basal $G_{t} s$ in the distal colon and rectum from the STD-F-fed group tended to be higher $(p<0.10)$ than that from the RRC-fed group.

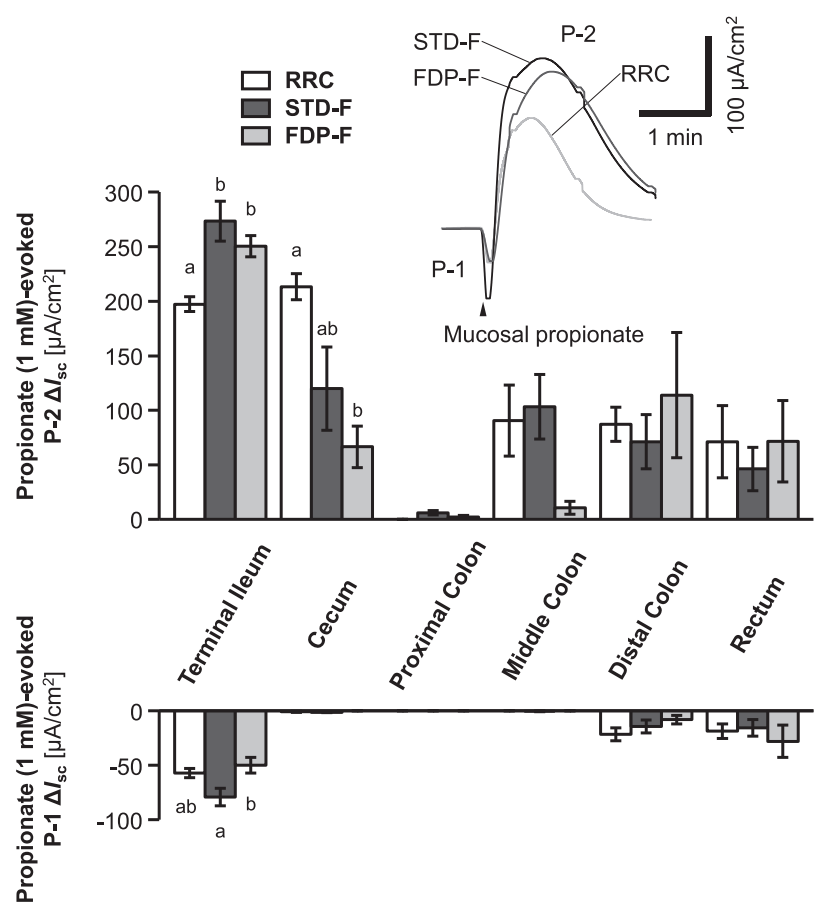

Fig. 3. Effects of RRC, STD-F and FDP-F feedings on the propionate-evoked changes in $I_{\mathrm{sc}}$ in mucosa-submucosal preparations of each segment of rat intestines. Propionate $\left(10^{-3} \mathrm{M}\right)$ was added to mucosal bath solution $1 \mathrm{~h}$ after EFS, and the propionate-evoked first decrease (P-1) and second increase (P-2) in $I_{s c}$ was determined. Data were expressed as mean \pm SE $(n=5-6)$. Different superscript alphabets differ significantly $(p<0.05)$ by Tukey-HSD or Games-Howell test. Inset traces: Stacks of representative traces of mucosal propionate-evoked $\Delta I_{\text {sc }}$ in each groups in the terminal ileum.

EFS-evoked increase in $I_{s c}$ in the intestinal mucosa-submucosa preparations

During the EFS, $I_{\mathrm{sc}}$ gradually increased and achieved maximum approximately until $2 \mathrm{~min}$ in all the segments, and returned to the basal level for several minutes after the EFS was completed as shown in Fig. 1 EFS). In the cecum and middle colon, the EFS-evoked $I_{\mathrm{sc}}$ response in the FDP-F-fed rat cecum was significantly reduced in comparison with the RRC- and STD-F-fed groups (Fig. 2, cecum and middle colon). In the proximal colon, the EFS-evoked $I_{\mathrm{sc}}$ response in both the STDF- and FDP-F-fed groups was significantly smaller than that in RRC-fed group (Fig. 2, proximal colon). In other segments, the EFS-evoked increases in $I_{\mathrm{sc}}$ were not significantly different among the feeding groups.

Mucosal propionate-evoked changes in $I_{s c}$

The mucosal addition of one of the SCFAs, propionate (1 mM), which was reported to evoke an increase in $I_{\mathrm{sc}}$ only at the mucosal side in the rat colon (28), evoked changes in $I_{\mathrm{sc}}$ except in the proximal colon (Fig. 1, $\boldsymbol{\Delta}$ propionate). Propionate evoked a fast-phasic negative change in $I_{\mathrm{sc}}$ within several seconds $(\mathrm{P}-1)$ in the terminal ileum, distal colon, and rectum, but not in the cecum and middle colon, and then a phasic positive change in $I_{\mathrm{sc}}$ in a few minutes (P-2) in all segments except the 
Table 3. Organic acid concentrations [mM] in cecal contents isolated from RRC-, STD-F- and FDP-F-fed rats.

\begin{tabular}{|c|c|c|c|}
\hline & $\mathrm{RRC}$ & STD-F & FDP-F \\
\hline \multicolumn{4}{|l|}{ SCFAs } \\
\hline Acetate & $91.0 \pm 4.6^{\mathrm{a}}$ & $53.0 \pm 5.1^{b}$ & $78.1 \pm 5.5^{\mathrm{a}}$ \\
\hline Propionate & $19.3 \pm 0.9^{\mathrm{a}}$ & $33.0 \pm 2.4^{\mathrm{b}}$ & $19.4 \pm 4.0^{\mathrm{a}}$ \\
\hline Butyrate & $52.7 \pm 1.5^{\mathrm{a}}$ & $9.2 \pm 0.8^{\mathrm{b}}$ & $21.3 \pm 1.5^{\mathrm{c}}$ \\
\hline Valerate & $3.2 \pm 0.2^{\mathrm{a}}$ & $1.7 \pm 0.5^{\mathrm{ab}}$ & $0.0 \pm 0.0^{b}$ \\
\hline Other organic acids & $0.2 \pm 0.0$ & $0.3 \pm 0.0$ & $1.7 \pm 0.7$ \\
\hline Formate & $0.2 \pm 0.0$ & $0.3 \pm 0.0$ & $1.7 \pm 0.7$ \\
\hline Lactate & $0.3 \pm 0.0$ & $0.3 \pm 0.1$ & $8.3 \pm 5.7$ \\
\hline Succinate & $0.3 \pm 0.01$ & $0.0 \pm 0.0$ & $3.0 \pm 2.8$ \\
\hline Total SCFAs & $166.2 \pm 3.4^{\mathrm{a}}$ & $96.8 \pm 5.1^{b}$ & $118.8 \pm 10.8^{b}$ \\
\hline Total organic acids & $166.9 \pm 3.4^{\mathrm{a}}$ & $97.5 \pm 5.2^{b}$ & $131.8 \pm 7.0^{c}$ \\
\hline
\end{tabular}

Data are expressed as mean \pm SE $(n=3)$. Different superscript alphabets differ significantly $(p<0.05)$ by Tukey test (acetate, propionate, butyrate, total SCFAs and total organic acids) or Games-Howells test (valerate).

Table 4. Bacterial densities in wet cecal contents isolated from RRC-, STD-F- and FDP-F-fed rats.

\begin{tabular}{lccc}
\hline \multicolumn{1}{c}{$(\log / \mathrm{g})$} & RRC & STD-F & FDP-F \\
\hline Bifidobacterium & $7.10 \pm 0.15^{\mathrm{a}}$ & $7.56 \pm 0.25^{\mathrm{a}}$ & $10.37 \pm 0.09^{\mathrm{b}}$ \\
Lactobacillus & $9.39 \pm 0.14$ & $9.51 \pm 0.06$ & $9.62 \pm 0.28$ \\
Bacteroides fragilis group & $7.85 \pm 0.11^{\mathrm{a}}$ & $10.28 \pm 0.10^{\mathrm{b}}$ & $10.02 \pm 0.23^{\mathrm{b}}$ \\
Clostridium coccoides group & $9.96 \pm 0.01^{\mathrm{a}}$ & $10.42 \pm 0.12^{\mathrm{ab}}$ & $10.81 \pm 0.21^{\mathrm{b}}$ \\
Clostridium leptum subgroup & $8.83 \pm 0.08^{\mathrm{a}}$ & $10.25 \pm 0.07^{\mathrm{b}}$ & $9.28 \pm 0.60^{\mathrm{ab}}$ \\
Enterobacteriaceae & $6.80 \pm 0.28^{\mathrm{a}}$ & $8.04 \pm 0.17^{\mathrm{ab}}$ & $8.48 \pm 0.56^{\mathrm{b}}$ \\
Enterococci & $7.15 \pm 0.24^{\mathrm{a}}$ & $7.81 \pm 0.15^{\mathrm{a}}$ & $8.73 \pm 0.11^{\mathrm{b}}$ \\
Total bacteria & $10.43 \pm 0.03^{\mathrm{a}}$ & $11.26 \pm 0.02^{\mathrm{b}}$ & $11.44 \pm 0.09^{\mathrm{b}}$ \\
\hline
\end{tabular}

Data are expressed as mean \pm SE $(n=3)$. Different superscript alphabets differ significantly $(p<0.05)$ by Tukey test (Bifidobacterium, Bacteroides fragilis group, Clostridium coccoides group, Enterobacteriaceae, and Enterococci) or Games-Howells test (Clostridium leptum subgroup and total bacteria).

proximal colon.

In the terminal ileum of the STD-F-fed group, the mucosal propionate-evoked P-1 $I_{\mathrm{sc}}$ response tended to be enhanced ( $p<0.10$; Fig. 3B terminal ileum), and the $\mathrm{P}-2$ response was significantly enhanced compared with the RRC-fed group (Fig. 3A terminal ileum). Although the $\mathrm{P}-2$ response in the FDP-F-fed group was also significantly enhanced (Fig. 3A terminal ileum), the P-1 response was not significantly different compared with the RRC-fed group (Fig. 3B terminal ileum).

In the cecum, although the propionate-evoked P-2 $I_{\mathrm{sc}}$ response in the STD-F-fed group was not significantly reduced compared with the RRC-fed group, the response in the FDP-F-fed group was significantly reduced compared with the RRC-fed group (Fig. 3A cecum).

In the middle colon, the propionate-evoked P-1 negative $I_{\mathrm{sc}}$ response in the STD-F-fed group tended to be reduced compared with the RRC-fed rats $(p<0.10$; Fig. 3B middle colon) although the responses were much smaller than the other segments. The P-2 $I_{\text {sc }}$ response in the FDP-F-fed group tended to be reduced compared with the STD-F-fed group $(p<0.10$; Fig. $3 A)$. In the other segments, the propionate-evoked increases in $I_{\mathrm{sc}}$ were not significantly different with each other.
Serosal CCh-evoked increase in $I_{s c}$

CCh $\left(10^{-5} \mathrm{M}\right)$, which is an analog of acetylcholine (ACh), the predominant secretagogue in the intestinal glands, evoked an increase in $I_{\text {sc }}$ in all preparations (Fig. $1, \triangle)$, and no significant difference was found among the feeding groups (Fig. S1 (Supplemental Online Material)). Concentrations of cecal organic acids

Organic acid concentrations in cecal contents are shown in Table 3. The total organic acid concentration of cecal contents in the STD-F-fed group was significantly reduced compared with the RRC- and STD-F-fed groups. Although the total organic acid concentration in the FDP-F-fed group was significantly lower than that in the RRC-fed group, the concentration in the FDPF-fed group was significantly higher than that in the STD-F-fed group. The total SCFA concentrations of cecal contents both in the STD-F- and FDP-F-fed groups were significantly reduced compared with the RRC-fed group. The butyrate and acetate concentrations in the STDF-fed group were significantly reduced compared with those in the RRC-fed group, but these concentrations in the STD-F-fed group were significantly increased compared with the FDP-F-fed group. Although the butyrate concentration in the FDP-F-fed group was significantly 
reduced compared with the RRC-fed group, it was significantly increased compared with the SDT-F-fed group. The acetate concentration in the SDT-F-fed group was also reduced compared with that in the RRC- and FDP-Ffed groups, but the concentration in the FDP-F-fed group was not significantly different from that in the RRC-fed group. On the other hand, the propionate concentration in the STD-F-fed group was significantly increased compared with the RRC- and FDP-F-fed groups, and no significant differences were found between the RRC- and FDP-F-fed groups.

Densities of cecal microbiota

Bacterial densities of cecal contents isolated from each rat group are shown in Table 4 . The total bacterial density in the STD-F- and FDP-F-fed groups was significantly higher than that in the RRC-fed group. In the STD-F-fed group, the densities of Bacteroides fragilis group and $C$. leptum subgroup were significantly higher than those in the RRC-fed group. In the FDP-F-fed group, the density of Bifidobacterium was significantly higher than that in the RRC- and STD-F-fed groups.

\section{DISCUSSION}

The present study showed that the consumption of STD-F enhances mucosal stimulant-mediated transepithelial ion transport in compared with RRC in terminal ileum of rats. This reason is unclear, but it might be due that RRC was composed of crude materials like crude grain in compared that STD-F was composed of purified materials. Whereas, FDP-F attenuated the enhancement, moreover FDP-F further attenuated the nervous system-mediated ion transport in cecum. It has been suggested that the inhibitory effects of FDP-F on the intestinal ion transport is possibly due to the changes in the components of gut microbiota and organic acids. These findings suggest that fermented dairy productand GOS-containing EF might possibly prevent the incidence of diarrhea during EN.

Effects of diets on electrophysiological parameters

The basal conditions of electrogenic transepithelial ion transport in all intestinal segments appear not to be changed by the diets, because basal $I_{\mathrm{sc}} \mathrm{s}$ in all intestinal segments were not significantly different among treatments (Table 2). Therefore, the cause of diarrhea during EN was suggested not to be attributed to the change in basal transepithelial ion transport. On the other hand, basal $G_{t} s$ in the cecum, middle colon, and distal colon were significantly higher than the others only in the FDP-F-fed group (Table 2). The reason is unknown, but it is indicated to be artificially due to the preparation process of removing contents, which were stickier in the FDP-F-fed group than in the other groups. The mucus layer of the FDP-F-fed rat might be removed more than that of the other groups.

Electrical field stimulation (EFS) to the intestinal mucosa-submucosa preparation activates submucosal and mucosal neurons of the enteric nervous system (ENS), inducing transepithelial anion secretion (29). The EFS-evoked secretory response has been reported to be mediated via ACh and other secretagogue neurotrans- mitters, predominantly vasoactive intestinal polypeptide (VIP) $(30,31)$. ACh and VIP directly activate epithelial cells, increase intracellular $\mathrm{Ca}^{2+}$ and cAMP, respectively, and then induce anion secretion. The present results suggest that FDP-F-feeding affects the ENS to reduce the intestinal secretion in the cecum and middle colon (Fig. 2 ), but not reducing that of the epithelial cells, because the direct activation of the epithelium by CCh (Fig. S1) was not affected by the dietary treatment.

Luminal propionate is reported to activate intestinal epithelial cells, releasing ACh from the epithelial cells, and activating neighboring epithelial cells and sensory nerve terminals (32). This induces epithelial secretory response mediated partially through the neural and partially through the non-neural direct pathways (28). The propionate-evoked negative $(\mathrm{P}-1)$ and positive $(\mathrm{P}-2)$ changes in $I_{\mathrm{sc}}$ are due to potassium $\left(\mathrm{K}^{+}\right)$and anion $\left(\mathrm{Cl}^{-}\right.$ and $\mathrm{HCO}_{3}{ }^{-}$) secretions, respectively (33). The transepithelial $\mathrm{K}^{+}$and anion secretions contribute in generating the driving force of epithelial fluid secretion. In the present study, STD-F-feeding enhanced the propionateevoked P-2 change in $I_{\mathrm{sc}}$ (anion secretion) in the terminal ileum compared with RRC-feeding (Fig. 3, terminal ileum). The terminal ileum is a segment that absorbs the most water in the intestine, so the enhancement of water secretion in the terminal ileum might possibly induce diarrhea. The cause of diarrhea during EN is possibly due to the higher sensitivity to luminal propionate. On the other hand, the propionate-evoked P-1 change in $I_{\mathrm{sc}}\left(\mathrm{K}^{+}\right.$secretion) in the FDP-F-fed group was significantly reduced compared with the STD-F-fed group (Fig. 3, terminal ileum). Moreover, in the FDP-F-fed group, the propionate-evoked P-1 change in $I_{\mathrm{sc}}$ (anion secretion) in the cecum was significantly reduced compared with the RRC-fed group (Fig. 3, cecum), suggesting the prevention of massive fluid secretion in the FDP-Ffed group. It is therefore suggested that STD-F-feeding makes the epithelium more sensitive to luminal propionate. These results suggest that FDP-F-feeding may reduce propionate-evoked fluid secretion by decreasing the sensitivity to propionate in the ileal and cecal epithelia and by inhibitory regulation of ENS in the cecum. Analysis of cecal organic acids and microbiota

It is unclear whether the changes in the electrophysiological activities of intestinal epithelia are regulated by the cecal organic acid concentrations and microbiota. However, the decrease in the luminal total organic acid concentration in both STD-F and FDP-F might induce an increase in the sensitivity of epithelial cells to propionate in the terminal ileum. In the FDP-F-fed group, the concentrations of acetate and butyrate significantly increased, whereas the propionate decreased compared with the STD-F-fed group (Table 3). These differences between the STD-F- and FDP-F-fed groups were possibly involved in the differences in the propionate-evoked responses, but this is unclear given the present results. Further studies are warranted in the future.

In the present analysis of cecal microbiota, the total bacterial densities in the cecal contents in the STD-Ffed and FDP-F-fed groups increased compared with the 
RRC-fed group (Table 4), although the total organic acid concentration in the STD-F-fed and FDP-F-fed groups was decreased (Table 3). The density of Bifidobacterium in FDP-F was significantly increased (Table 4), suggesting that the increase in Bifidobacterium might contribute to the increase in acetate and butyrate.

Length of the intestines

Colonic mucosal atrophy is induced by liquid elemental diet in rats (34), and epithelial proliferation is decreased in the colon of rats fed a liquid diet (35). Although I did not perform histological analysis of mucosa, but measured the lengths of small and large intestines. The results showed that the length of large intestine was shorten in the STD-F-fed rats, but not in the FDP-F-fed rats compared to the RRC-fed rats (Table 1).

SCFAs, especially butyrate, are known as a trophic substance to the intestinal mucosa $(36,37)$. The trophic effect of FDP-F is suggested to be possibly due to the increase in SCFA, especially butyrate production in the large intestine. We previously have reported that the SCFA receptors, FFA2 (GPR43) and FFA3 (GPR41), are expressed in the intestinal epithelium, particularly peptide YY (PYY) and/or glucagon-like peptide 1 (GLP1) containing L-type enteroendocrine cells in the ileum and colon of humans and rats (38-40). GLP-2, which is produced from the same precursor with GLP-1, is known to have a trophic effect that is highly specific for the GI tract (41). In addition, it has been reported that GLP-2 increases a length of small intestine in parenterally fed rats with short bowel syndrome (42). Therefore, it is suggested that the increase in luminal SCFA enhances GLP-2 endocrine, inducing the increase in the length of the large intestine.

Conclusions

The present study showed that STD-F consumption increased the sensitivity for luminal propionate. Moreover, FDP-F consumption decreased the secretory nerve activity in the cecum, proximal colon, and middle colon, and the sensitivity for propionate in the cecum. These might be due to the total amounts and components of gut microbiota and organic acids, and inhibitory changes in mucosal luminal stimulant- and nervous system-mediated fluid secretory function.

\section{Disclosure of state of COI}

This study was partially funded and supported in personnel and materials by Meiji Co., Ltd.

\section{Acknowledgments}

This study was partially funded by JSPS KAKENHI Grant-in-Aid for Scientific Research (C) to S. Karaki (No. 17K08570).

\section{Supporting information}

Supplemental online material is available on J-STAGE.

\section{REFERENCES}

1) Boullata JI, Carrera AL, Harvey L, Escuro AA, Hudson L, Mays A, McGinnis C, Wessel JJ, Bajpai S, Beebe ML,
Kinn TJ, Klang MG, Lord L, Martin K, Pompeii-Wolfe C, Sullivan J, Wood A, Malone A, Guenter P, ASPEN Safe Practices for Enteral Nutrition Therapy Task Force; American Society for Parenteral and Enteral Nutrition. 2017. ASPEN Safe Practices for Enteral Nutrition Therapy. JPEN J Parenter Enteral Nutr 41: 15-103.

2) Whelan K. 2007. Enteral-tube-feeding diarrhoea: manipulating the colonic microbiota with probiotics and prebiotics. Proc Nutr Soc 66: 299-306.

3) Eisenberg P. 2002. An overview of diarrhea in the patient receiving enteral nutrition. Gastroenterol Nurs 25: 95-104.

4) Whelan K, Judd PA, Preedy VR, Taylor MA. 2004. Enteral feeding: the effect on faecal output, the faecal microflora and SCFA concentrations. Proc Nutr Soc 63: 105-113.

5) Hill C, Guarner F, Reid G, Gibson GR, Merenstein DJ, Pot B, Morelli L, Canani RB, Flint HJ, Salminen S, Calder PC, Sanders ME. 2014. Expert consensus document. The International Scientific Association for Probiotics and Prebiotics consensus statement on the scope and appropriate use of the term probiotic. Nat Rev Gastroenterol Hepatol 11: 506-514.

6) Roberfroid M. 2007. Prebiotics: the concept revisited. J Nutr 137: 830S-837S.

7) Whelan K, Gibson GR, Judd PA, Taylor MA. 2001. The role of probiotics and prebiotics in the management of diarrhoea associated with enteral tube feeding. J Hum Nutr Diet 14: 423-433.

8) Ferrie S, Daley M. 2011. Lactobacillus GG as treatment for diarrhea during enteral feeding in critical illness: randomized controlled trial. JPEN J Parenter Enteral Nutr 35: 43-49.

9) Bleichner G, Bléhaut H, Mentec H, Moyse D. 1997. Saccharomyces boulardii prevents diarrhea in critically ill tube-fed patients. A multicenter, randomized, doubleblind placebo-controlled trial. Intensive Care Med 23: 517-523.

10) Elia M, Engfer MB, Green CJ, Silk DB. 2008. Systematic review and meta-analysis: the clinical and physiological effects of fibre-containing enteral formulae. Aliment Pharmacol Ther 27: 120-145.

11) Kamarul Zaman M, Chin KF, Rai V, Majid HA. 2015. Fiber and prebiotic supplementation in enteral nutrition: A systematic review and meta-analysis. World J Gastroenterol 21: 5372-5381.

12) Majid HA, Cole J, Emery PW, Whelan K. 2014. Additional oligofructose/inulin does not increase faecal bifidobacteria in critically ill patients receiving enteral nutrition: a randomised controlled trial. Clin Nutr 33: 966-972.

13) Metchnikoff É. 1908. The Prolongation of Life: Optimistic Studies (Mitchell PC, ed). The Knickerbocker Press, New York.

14) Tamime AY, Robinson RK. 2007. Microbiology of yoghurt and related starter cultures. In: Tamime and Robinson's Yoghurt, 3rd ed, p 468-534. Woodhead Publishing, Cambridge.

15) Satomi K, Kurihara H, Isawa K, Mori H, Kaneko T. 1999. Effects of culture-powder of Propionibacterium freudenreichii ET-3 on fecal microflora of normal adults. Biosci Microflora 18: 27-30.

16) Isawa K, Hojo K, Yoda N, Kamiyama T, Makino S, Saito M, Sugano H, Mizoguchi C, Kurama S, Shibasaki M, Endo N, Sato Y. 2002. Isolation and identification of a 
new bifidogenic growth stimulator produced by Propionibacterium freudenreichii ET-3. Biosci Biotechnol Biochem 66: 679-681.

17) Bowling TE, Raimundo AH, Grimble GK, Silk DB. 1993. Reversal by short-chain fatty acids of colonic fluid secretion induced by enteral feeding. Lancet 342: 1266-1268.

18) Karaki S, Kuwahara A. 2004. Electrophysiological measurement of transepithelial ion transport: short-circuit current (Ussing chamber) technique. Nihon Yakurigaku Zasshi 123: 211-218.

19) Karaki S, Ishikawa J, Tomizawa Y, Kuwahara A. 2016. Effects of $\varepsilon$-viniferin, a dehydrodimer of resveratrol, on transepithelial active ion transport and ion permeability in the rat small and large intestinal mucosa. Physiol Rep 4: e12790.

20) Kaneko T, Mori H, Iwata M, Meguro S. 1994. Growth stimulator for bifidobacteria produced by Propionibacterium freudenreichii and several intestinal bacteria. J Dairy Sci 77: 393-404.

21) Niwa T, Nakao M, Hoshi S, Yamada K, Inagaki K, Nishida M, Nabeshima T. 2002. Effect of dietary fiber on morphine-induced constipation in rats. Biosci Biotechnol Biochem 66: 1233-1240.

22) Nagafuchi S, Yamaji T, Kawashima A, Saito Y, Takahashi T, Yamamoto T, Maruyama M, Akatsu H. 2015. Effects of a formula containing two types of prebiotics, bifidogenic growth stimulator and galacto-oligosaccharide, and fermented milk products on intestinal microbiota and antibody response to influenza vaccine in elderly patients: a randomized controlled trial. Pharmaceuticals (Basel) 8: 351-365.

23) Akatsu H, Nagafuchi S, Kurihara R, Okuda K, Kanesaka T, Ogawa N, Kanematsu T, Takasugi S, Yamaji T, Takami M, Yamamoto T, Ohara H, Maruyama M. 2016. Enhanced vaccination effect against influenza by prebiotics in elderly patients receiving enteral nutrition. Geriatr Gerontol Int 16: 205-213.

24) Matsuki T, Watanabe K, Fujimoto J, Takada T, Tanaka R. 2004. Use of $16 \mathrm{~S}$ rRNA gene-targeted group-specific primers for real-time PCR analysis of predominant bacteria in human feces. Appl Environ Microbiol 70: 7220-7228.

25) Dubernet S, Desmasures N, Gueguen M. 2002. A PCRbased method for identification of lactobacilli at the genus level. FEMS Microbiol Lett 214: 271-275.

26) Bartosch S, Fite A, Macfarlane GT, McMurdo ME. 2004. Characterization of bacterial communities in feces from healthy elderly volunteers and hospitalized elderly patients by using real-time PCR and effects of antibiotic treatment on the fecal microbiota. Appl Environ Microbiol 70: 3575-3581.

27) Rinttilä T, Kassinen A, Malinen E, Krogius L, Palva A. 2004. Development of an extensive set of 16S rDNAtargeted primers for quantification of pathogenic and indigenous bacteria in faecal samples by real-time PCR. J Appl Microbiol 97: 1166-1177.
28) Yajima T. 1998. Luminal propionate-induced secretory response in the rat distal colon in vitro. J Physiol 403: 559-575.

29) Cooke HJ, Shonnard K, Wood JD. 1983. Effects of neuronal stimulation on mucosal transport in guinea pig ileum. Am J Physiol Gastrointest Liver Physiol 245: G290-G296.

30) Cooke HJ. 2000. Neurotransmitters in neuronal reflexes regulating intestinal secretion. Ann NY Acad Sci 915: 77-80.

31) Karaki SI, Kuwahara A. 2004. Regulation of intestinal secretion involved in the interaction between neurotransmitters and prostaglandin $\mathrm{E}_{2}$. Neurogastroenterol Motil 16 (Suppl 1): 96-99.

32) Yajima T, Inoue R, Matsumoto M, Yajima M. 2011. Nonneuronal release of ACh plays a key role in secretory response to luminal propionate in rat colon. J Physiol 589: 953-962.

33) Karaki S, Kuwahara A. 2011. Propionate-induced epithelial $\mathrm{K}^{+}$and $\mathrm{Cl}^{-} / \mathrm{HCO}_{3}{ }^{-}$secretion and free fatty acid receptor 2 (FFA2, GPR43) expression in the guinea pig distal colon. Pflugers Arch 461: 141-152.

34) Janne P, Carpentier Y, Willems G. 1977. Colonic mucosal atrophy induced by a liquid elemental diet in rats. Am J Dig Dis 22: 808-812.

35) Storme G, Willems G. 1981. The effect of a liquid elemental diet on cell proliferation in the colon of rats. Cell Tissue Res 216: 221-225.

36) Sakata T. 1987. Stimulatory effect of short-chain fatty acids on epithelial cell proliferation in the rat intestine: a possible explanation for trophic effects of fermentable fibre, gut microbes and luminal trophic factors. Br J Nutr 58: $95-103$.

37) Guilloteau P, Martin L, Eeckhaut V, Ducatelle R, Zabielski R, Van Immerseel F. 2010. From the gut to the peripheral tissues: the multiple effects of butyrate. Nutr Res Rev 23: 366-384.

38) Karaki S, Mitsui R, Hayashi H, Kato I, Sugiya H, Iwanaga T, Furness JB, Kuwahara A. 2006. Short-chain fatty acid receptor, GPR43, is expressed by enteroendocrine cells and mucosal mast cells in rat intestine. Cell Tissue Res 324: 353-360.

39) Karaki S, Tazoe H, Hayashi H, Kashiwabara H, Tooyama K, Suzuki Y, Kuwahara A. 2008. Expression of the shortchain fatty acid receptor, GPR43, in the human colon. J Mol Histol 39: 135-142.

40) Tazoe H, Otomo Y, Karaki S, Kato I, Fukami Y, Terasaki M, Kuwahara A. 2009. Expression of short-chain fatty acid receptor GPR41 in the human colon. Biomed Res 30: 149-156.

41) Burrin DG, Petersen Y, Stoll B, Sangild P. 2001. Glucagon-like peptide 2: a nutrient-responsive gut growth factor. J Nutr 131: 709-712.

42) Martin GR, Wallace LE, Sigalet DL. 2004. Glucagon-like peptide- 2 induces intestinal adaptation in parenterally fed rats with short bowel syndrome. Am J Physiol Gastrointest Liver Physiol 286: G964-G972. 